\title{
속보 (Communications)
}

\section{A Photoactive Antimicrobial Triphenylmethane Derivative}

\author{
Jung Lee, Kyung Wha $\mathrm{Oh}^{\dagger, *}$, and Jong-Man Kim* \\ Department of Chemical Engineering, Hanyang University, Seoul 133-791, Korea \\ 'Department of Home Economics Education, Chung-Ang University, Seoul 156-756, Korea. \\ EE-mail:jmk@hanyang.ac.kr,kwhaoh@cau.ac.kr \\ (Received March 15, 2012; Accepted March 19, 2012)
}

Key words: Antimicrobial agent, Photoactive, Triphenylmethane

Owing to the ever growing interest for the human health against infectious bacteria, various substances that display antimicrobial activity have been developed. ${ }^{1-7}$ For instance, inorganic silver nanoparticles have been actively investigated as antibacterial agents although the mechanism for the activity is not clearly understood..$^{8-12}$

Recently, photochemically triggerable organic antimicrobial substances have received significant attention since these materials can be formulated with fabrics to produce self-decontaminating clothes. ${ }^{13-18}$ In addition, the irradiation-induced antimicrobial activity is attractive because of the readily available light source (eg. Sun). Among various candidates, benzophenone derivatives have been most actively investigated as photoactive antimicrobial agents owing to the some salient features including facile synthesis and generation of reactive radicals via photosensitization. ${ }^{13-15}$ Thus, benzoquinone with diverse substituents have been prepared and applied to the antimicrobial screening.

It has been well known that photoinduced oxidation of certain electron donating group-substituted triphenylmethane derivatives produces brilliantly colored ionic species. ${ }^{19}$ Owing to this property, triphenylmethane derivatives have been investigated as precursors of colorants. A mechanistic pathway to the photochemical genartion of an ionic iminium product from a triphenyl methane drivative, 4,4',4"-tris(dimethylaminophenyl) methane (TPM, 1) is presented in Scheme 1. The first step of this process involves excited state single electron transfer (SET) from the triphenylmethane donor to presumably molecular oxygen. The radical cation intermediate 2 , produced in this fashion, subsequently undergoes hydrogen atom loss to yield the iminium ion $\mathbf{3}$. Since the iminium ion $\mathbf{3}$ is fully conjugated, formation of the ionic moiety can be readily monitored by visible absorption spectroscopy and most often with naked eyes.
The generation of reactive ionic/radical species from the photochemical oxidation of TPM is very intriguing since these reactive species might be able to retard the growth of microbes or destroy them. If TPM displays the antimicrobial activity, a new class of photoactive antimicrobial agent can be developed.

In order to test the feasibility of TPM as a photoactive antimicrobial agent, a thin (ca. $1 \mathrm{~m}$ ) polystyrene (PS) film containing TPM was prepared by spin-coating of a chloroform solution containing PS (MW: 280,000, $5 \mathrm{wt} \%$ ) and TPM (1.5 wt $\%$, based on the weight of PS polymer powder) on a glass substrate. ${ }^{20}$ Irradiation of UV light (254 $\mathrm{nm}, 1 \mathrm{~mW} / \mathrm{cm}^{2}$ ) to the film resulted in the gradual increase of the absorption in the visible region, confirming the successful generation of iminium product $\mathbf{3}$ in the polymer film (Fig. 1). In addition, the colorless transparent TMPcontaining film became pale purple after UV irradiation.

We next investigated antimicrobial properties of the TPM containing PS films against Staphylococcus aureus
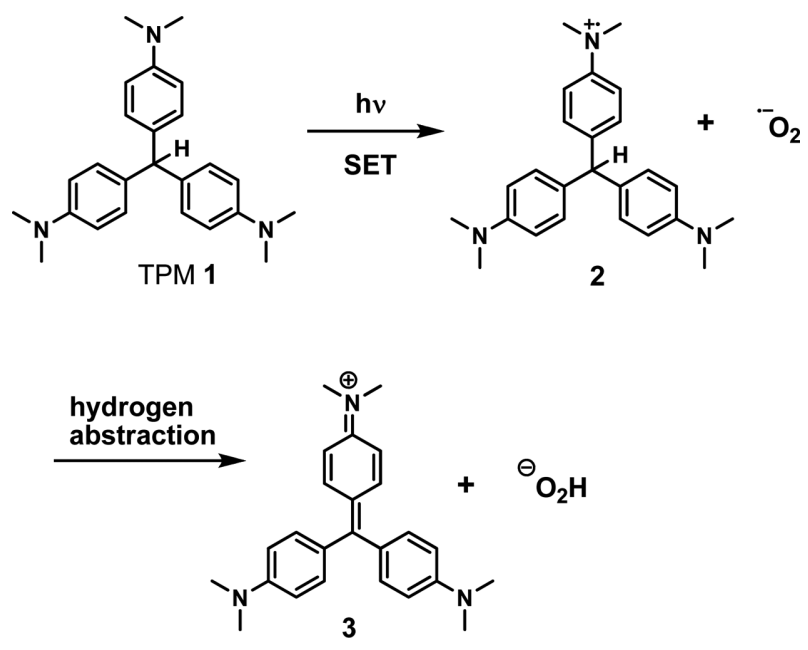

Scheme 1. Proposed mechanism of photoinducediminium ion formation from TPM. 


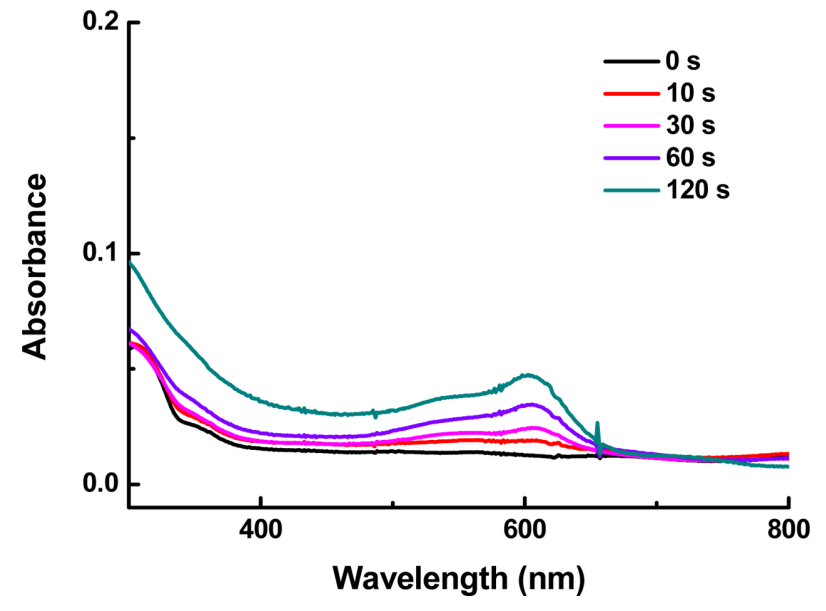

Fig. 1. UV-visible absorption spectral change obtained with PS film containing TPM upon UV irradiation.

(ATCC 6538P) according to the method of contact film (JIS Z 2801). ${ }^{21,22}$ A $0.2 \mathrm{~mL}$ of diluted microbe solution (stock microbe solution: $2.1 \sim 2.5 \times 10^{5} \mathrm{CFU} / \mathrm{mL}$ ) was applied to a TPM-containing PS film $(5 \times 5 \mathrm{~cm})$ in a Petri dish. A control experiment was carried out with a PS film without TPM. The resultant Petri dish was irradiated with UV light $\left(254 \mathrm{~nm}, 1 \mathrm{~mW} / \mathrm{cm}^{2}\right)$ for $2 \mathrm{~h}$. After UV irradiation, the microbes in Petri dish was collected with a 20 $\mathrm{mL}$ distilled water and $1 \mathrm{~mL}$ of the solution was transferred to a test tube of $9 \mathrm{~mL}$ saline solution. Then, $1 \mathrm{~mL}$ of the diluted solution was spread on a new Petri dish with a growth medium of trypticase soy agar. The plate was incubated at $37^{\circ} \mathrm{C}$ for $24 \mathrm{~h}$ and the number of colonies was determined. The antibicrobial activity was evaluated using the following equations.

Antimicrobial activity $(\mathrm{S})=\log \frac{\mathrm{Mc}}{\mathrm{Ma}}-\log \frac{\mathrm{Mc}}{\mathrm{Ma}}=\log \frac{\mathrm{Mb}}{\mathrm{Mc}}$

$$
\text { Degree of reduction }(\%)=\frac{M b-M c}{M b} \times 100
$$

where, Ma is an average number of bacteria in reference sample immediately after vaccination of testing bacteria, $\mathrm{Mb}$ is an average number of bacteria in reference sample after incubation for $24 \mathrm{~h}$, and $\mathrm{Mc}$ is an average number of bacteria in antimicrobial sample after incubation for $24 \mathrm{~h}$.

Table 1 shows antimicrobial functions of the pristine PS film and the TPM (1.5 wt \%, based on the weight of PS polymer powder) containing PS films. The number of colonies of Staphylococcus aureus were significantly reduced after UV irradiation of the TPM containing PS film (99.3\% reduction) compared to the nonirradiated counterpart (33.0\% reduction). In contrast, the control PS film which does not contain TPM molecules displayed a slight increase in the colony reduction ( 25.5 to $36.7 \%$ reduction). These results indicate that the UV irradiated TPM molecules are able to inhibit effectively the growth of the Staphylococcus aureus.

The UV-irradiated formation of iminium ion species from TPM was evidenced by observing color change of the Petri dish (Fig. 2). Accordingly, the Petri dish that con-
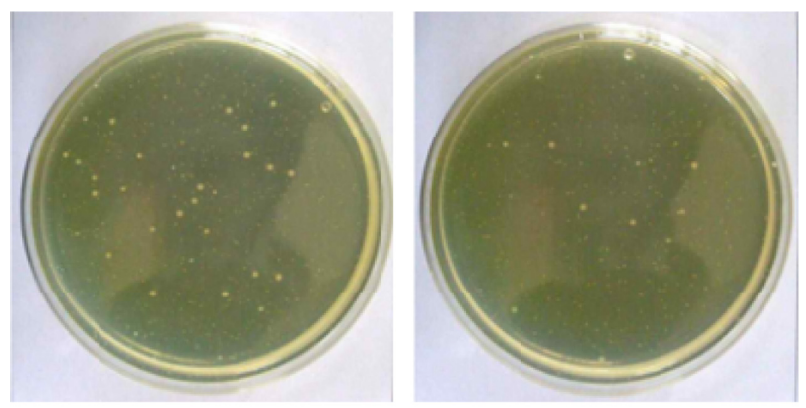

(a)
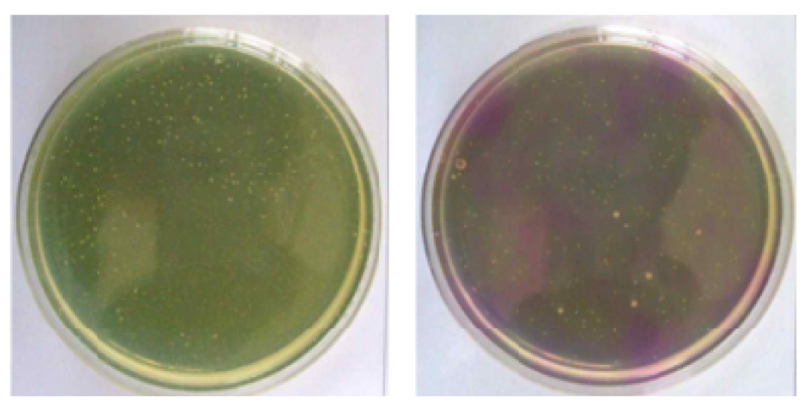

(b)

Fig. 2. Photographs of Petri dishes before (a) and after (b) UV irradiation $\left(254 \mathrm{~nm}, 1 \mathrm{~mW} / \mathrm{cm}^{2}, 2 \mathrm{~h}\right)$ of pristine PS (left) and TPM (1.5 wt\%)-containing PS (right) films in the presence of colonies of Staphylococcus aureus.

Table 1. Antimicrobial test results of pristine PS and TPM (1.5 wt \%) containing PS films after UV irradiation for $2 \mathrm{~h}$

\begin{tabular}{ccccccc}
\hline & & \multicolumn{5}{c}{ Staphylococcus aureus } \\
\cline { 3 - 6 } & & Ma & Mb & Mc & Antimicrobial activity (S) & Reduction (\%) \\
\hline \multirow{2}{*}{ Before UV } & Pristine PS & $2.2 \times 10^{5}$ & $2.7 \times 10^{5}$ & $2.0 \times 10^{5}$ & 0.1 & 25.5 \\
& TPM & $2.2 \times 10^{5}$ & $2.7 \times 10^{5}$ & $1.8 \times 10^{5}$ & 0.2 & 33.0 \\
\hline \multirow{2}{*}{ After UV } & Pristine PS & $2.6 \times 10^{5}$ & $3.1 \times 10^{5}$ & $2.0 \times 10^{5}$ & 0.2 & 36.7 \\
& TPM & $2.6 \times 10^{5}$ & $3.1 \times 10^{5}$ & $2.2 \times 10^{3}$ & 2.1 & 99.3 \\
\hline
\end{tabular}


tains only pristine PS film (Fig. 2a, left) did not show any color change after UV irradiation (Fig. 2b, left). In contrast, the generation of purple color was monitored (Fig. $2 b$, right) form the UV irradiation of the TPM-containing Petri dish (Fig. 2a, right).

It is unclear at this moment the exact mechanism of the photoinduced antimicrobial activity of TPM molecules. We, however, believe that the photochemically produced reactive species (either radical or ionic) are responsible for the inhibition of the bacteria growth.

In summary, a new class of photoactive antimicrobial agent was developed. The electron-donating group substituted triphenylmethane derivative TPM displayed good antibacterial activity upon UV irradiation. The UV-induced generation of iminium ions was observed with a TPM containing PS film. Regardless of the mechanistic details, the results described above should be considered as important contributions to the ever growing interest in the antibacterial research area.

Acknowledgements. This research was supported by the National Research Foundation of Korea (Project No. 2011-0004587).

\section{REFERENCES}

1. Dhende, V. P.; Samanta, S.; Jones, D. M.; Hardin, I. R.; Locklin, J. ACS Appl. Mater. Interfaces 2011, 3, 2830.

2. Volkmann, A.; Ghosh, S. J. Appl. Polym. Sci. 2011, 119, 1646.

3. James, C.; Johnson, A. L.; Jenkins, A. T. A. Chem. Commun. 2011, 47, 12777.

4. Rai, A.; Prabhune, A.; Perry, C. C. J. Mater. Chem. 2010, 20, 6789.

5. Malmsten, M. Soft Matter 2011, 7, 8725.

6. Wang, J.; Chen, Y. P.; Yao, K.; Wilbon, P. A.; Zhang, W.;
Ren, L.; Zhou, J.; Nagarkatti, M.; Wang, C.; Chu, F.; He, X.; Decho, A. W.; Tang, C. Chem. Commun. 2012, 48, 916.

7. Santos, C. M.; Tria, M. C. R.; Vergara, R. A. M. V.; Ahmed, F.; Advincula, R. C.; Rodrigues, D. F. Chem. Commun. 2011, 47, 8892.

8. Zhang, B.; Luo, Y.; Wang, Q. Biomacromolecules 2010 , 11, 2366.

9. Sambhy, V.; MacBride, M. M.; Peterson, B. R.; Sen, A. J. Am. Chem. Soc. 2006, 128, 9798.

10. Eby, D. M.; Luckarift, H. R.; Johnson, G. R. ACS Appl. Mater. Interfaces 2009, 1, 1553.

11. Wang, Y.; Cao, L.; Guan, S.; Shi, G.; Luo, Q.; Miao, L.; Thistlethwaite, I.; Huang, Z.; Xu, J.; Liu, J. J. Mater. Chem. 2012, 22, 2575.

12. Sureshkumar, M.; Siswanto, D. Y.; Lee, C.-K. J. Mater. Chem. 2010, 20, 6948.

13. Hong, K. W.; Sun, G. J. Appl. Polym. Sci. 2007, 106, 2661.

14. Hong, K. W.; Sun, G. J. Appl. Polym. Sci. 2008, 109, 3173.

15. Lim, K. S.; Oh, K. W. Textile Sci. Eng. 2011, 48, 42

16. Mizukami, S.; Hosoda, M.; Satake, T.; Okada, S.; Hori, Y.; Furuta, T.; Kikuchi, K. J. Am. Chem. Soc. 2010, 132, 9524.

17. Towers, A. G. H. N.; Rinehart, K. L. Photochem. Photbiol. 1988, 47, 377.

18. Perni, S.; Prokopovich, P.; Parkin, I. P.; Wilson, M.; Pratten, J. J. Mater. Chem. 2010, 20, 8668.

19. Malpert, J. H.; Grinevich, O.; Strehmel, B.; Jarikov, V.; Mejiritski, A.; Neckers, D. C. Tetrahedron 2001, 57, 967.

20. TPM is commercially available from Aldrich.

21. TPM containing PS films was fabricated following the modified procedure described in the literature (ref. 9). To a PS solution in chloroform (initial concentration $10 \mathrm{wt} \%$ ) was added a TPM solution (chloroform) at room temperature. The resultant mixture (98.5 PS and 1.5 TPM, wt $\%$ ratio) was casted on a Teflon sheet and dried in a fume hood at room temperature for $24 \mathrm{~h}$.

22. The antimicrobial activity of the TPM-containing PS film was carried out by Bioteca co, Ltd. 